\title{
Can preoperative findings help to interpret neoplastic and non-neoplastic lesions of ovary and affect surgical decisions in children and adolescents?
}

\author{
Asist. Prof. İdil R. User ${ }^{a}$, Assoc. Prof. Süleyman C. Karakuş̧, \\ Prof. Bülent H. Özokutan ${ }^{a}$, Vedat Akçaer, M.D. ${ }^{a}$, Bayram Burulday, M.D. ${ }^{a}$ \\ and Prof. Haluk Ceylan ${ }^{a}$
}

\begin{abstract}
Background: Ovarian masses are common in all pediatric age groups. Ovarian-sparing surgery is favored since most cases are benign or functional cysts. Detection of a few malignant cases prevents morbidity and mortality. Objective: to investigate which of the preoperative findings can help to distinguish the pathology of the ovarian lesions, affect the surgical decision and predict the possibility of ovarian preservation.

Method: Patients operated with diagnosis of ovarian mass were enrolled in the study between 2000-2015. Age, symptoms, physical examination findings, tumor markers, radiologic features, operative notes, and pathology results were reviewed retrospectively.

Results: During the study period, 98 patients were operated and 86 were included. Mean age was $9.7 \pm 5.62$ years. Non-neoplastic pathologies constitute $68.6 \%$ of cases; $31.4 \%$ was neoplastic. Malignancy rate was $4.6 \%$. Solid component, absence of ovarian torsion and diameter $>9 \mathrm{~cm}$ were more commonly seen in neoplastic cases $(\mathrm{p}<0.001, \mathrm{p}<0.001, \mathrm{p}=0.001)$. Adnexal torsion was found in $30(34.9 \%)$ patients. Mean diameter did not differ significantly between groups with or without torsion; incidence of torsion was greater in patients with masses $<6 \mathrm{~cm}$ ( $\mathrm{p}=0.019)$. Ovariansparing surgery was performed in $48(55.8 \%)$ and oopherectomy in $38(44.2 \%)$ patients. Surgical approach was affected by nature $(\mathrm{p}<0.001)$ and size $(p<0.001)$ of the lesion; it was independent of age and presence of torsion.

Conclusion: A purely cystic mass $<9 \mathrm{~cm}$, with torsion and negative markers, orients towards a non-neoplastic mass. The presence of solid component and absence of torsion are associated with increased risk of malignancy.
\end{abstract}

Key words: child, ovary, surgery, organ sparing treatments.

http:/ / dx.doi.org/10.5546/ aap.2019.eng.294

E-mail address:

Süleyman Cüneyt

Karakuş:

sckarakus@yahoo.com

Funding:

None.

Conflict of interest:

None.

Received: 1-23-2019

Accepted: 5-1-2019

\section{INTRODUCTION}

The ovarian masses are common in the infant-juvenile population; they are the most common genital neoplasms in childhood. Ovarian neoplasms (benign and malignant) account for approximately $1 \%$ of all tumors in children and adolescents. ${ }^{1}$ Since children have a long life span and expectancy, ovarian functions and fertility are important issues in this age group. These facts lead to a trend of ovarian-sparing surgery in both cystic and solid lesions in pediatric patients. ${ }^{2,3}$ However strong presumption of malignancy, destruction of ovarian tissue by the mass and necrosis caused by torsion may necessitate oopherectomy. ${ }^{2,3}$

The objective was to investigate which of the preoperative findings can help to distinguish the pathology of the ovarian lesions, affect the surgical decision and predict the possibility of ovarian preservation.

\section{POPULATION AND METHODS}

Our study included patients operated with the diagnosis of ovarian mass in our Pediatric Surgery Clinic between the years 2000-2015. Indications of surgical treatment were: symptoms attributed to mass, suspicion of malignancy, unresponsiveness to conservative management and presence of acute abdomen. All patients' files were reviewed retrospectively for age, symptoms, physical examination findings, tumor markers, radiologic features, operative notes, and pathology results. This study was approved by Local Ethics Committee for Clinical Investigations. 
Statistical analyses were done with SPSS ver.15.0 (SPSS Inc., Chicago, IL, USA). $\mathrm{p}<0.05$ was accepted as statistically significant. Consistency of normal data distribution was tested by Shaphirowilk test. Comparison of numerical data was done by Mann Whitney U test. Chi-square test was used to study the relation between categorical variables. ROC curve analysis was used for cut-off point calculation.

\section{RESULTS}

\section{a) Descriptive data}

A total of 98 girls aged between 0 and 16 years were treated in this time period but 86 of them were enrolled in the study; 12 were excluded because of lost patient charts and inadequate information. In 86 girls, there were 88 ovarian masses. Two patients had bilateral masses: a newborn with bilateral massive follicle cysts and an adolescent with simple serous cysts. Age distribution ranges from newborn to 16 years old and the mean age was $9.7 \pm 5.62$ years. Infants younger than 1 year of age constitute $17.4 \%$ of cases. Presenting symptoms were chronic abdominal pain $(\mathrm{n}=32,37.2 \%)$ and menstrual irregularity or dysmenorrhea $(n=16,18.6 \%)$. Six patients presented to the pediatrics clinic with symptoms of precocious puberty and further investigations revealed ovarian masses in these patients. In 12 patients diagnosis was done prenatally. Acute abdomen was detected in 23 $(26.7 \%)$ girls.

Tumor markers were studied in 25 patients in the preoperative period. AFP was elevated in 3 patients with the following diagnosis: mature cystic teratoma, dysgerminoma and immature teratoma. CA 19-9 was elevated in 4 patient in 2 cases of mature cystic teratoma, one of dermoid cyst and one of immature teratoma. CA-125 was elevated in mature cystic teratoma, dermoid cyst, immature teratoma and endometriosis in one patient of each diagnosis. None of the patients had elevated level of $\beta$-hcg. All cases with high marker levels had neoplastic pathologies except the girl with high CA-125 having endometriosis. Patients presenting with precocious puberty had follicular cyst $(n=3)$, serous cystadenoma $(n=2)$, juvenile granulosa cell tumor $(n=1)$. Elevated levels of estrogen were detected in these patients.

Right side was affected in $48(55.8 \%)$ and left in $36(41.9 \%)$ patients and bilateral in $2(2.3 \%)$. Mean greatest diameter of mass measured by USG was $8.25 \pm 4.59 \mathrm{~cm}$ (range 3-24 cm). USG revealed cystic mass in $57(66.3 \%)$, solid in $5(5.8 \%)$ and both cystic and solid components in $24(27.9 \%)$ patients. Surgical approach was laparoscopy in $16(18.6 \%)$ with ovary-sparing surgery in 15 patients, and laparotomy in $70(81.3 \%)$ with ovary-sparing surgery in 33 patients.

Adnexal torsion was encountered in 30 $(34.9 \%)$ cases. Mean diameter of ovaries with torsion was $7.08 \pm 3.73 \mathrm{~cm}$ and it was $8.8 \pm 4.9 \mathrm{~cm}$ in the others. This difference was not statistically significant $(p=0.069)$. However the incidence of torsion was lower in masses $>6 \mathrm{~cm}$ compared to smaller ones. The percentage of torsion was $25.5 \%$ and $51.6 \%$ respectively in each group and the difference was statistically significant $(\mathrm{p}=0.019)$. The lesion was purely cystic in $80 \%$ and cases with cystic and solid components constitute $20 \%$. Torsion was not detected in none of the pure solid lesions.

The histopathological diagnoses of nonneoplastic and neoplastic pathologies are stated in Table 1.

\section{b) Predictor parameters of pathology:} age, size, USG features, adnexal torsion (Table 2)

Patients were divided into 2 groups according to neoplastic and non-neoplastic pathologies. There is no statistically significant difference of age with respect to pathology group $(\mathrm{p}=0.470)$.

TABLE 1. Pathologic diagnosis

\begin{tabular}{ll} 
Non-neoplastic $(\mathrm{n}=59 ; 68.6 \%)$ & Neoplastic $(\mathrm{n}=27 ; 31.4 \%)$ \\
Follicle cyst $(\mathrm{n}=21 ; 24.4 \%)$ & Dermoid cyst $(\mathrm{n}=7 ; 8.1 \%)$ \\
Simple cyst $(\mathrm{n}=17 ; 19.7 \%)$ & Mature cystic teratoma $(\mathrm{n}=6 ; 7 \%)$ \\
Corpus luteum cyst $(\mathrm{n}=12 ; 13.9 \%)$ & Dysgerminoma $(\mathrm{n}=2 ; 2.3 \%)$ \\
Serous cyst $(\mathrm{n}=5 ; 5.8 \%)$ & Immature teratoma $(\mathrm{n}=2 ; 2.3 \%)$ \\
Hemorrhagic cyst $(\mathrm{n}=2 ; 2.3 \%)$ & Mucinous cystadenoma $(\mathrm{n}=3 ; 3.4 \%)$ \\
Endometriosis $(\mathrm{n}=1 ; 1.1 \%)$ & Mucinous borderline tumor $(\mathrm{n}=3 ; 3.4 \%)$ \\
Massive ovarian edema $(\mathrm{n}=1 ; 1.1 \%)$ & Serous papillary cystadenoma $(\mathrm{n}=3 ; 3.4 \%)$ \\
& Juvenile granulosa cell $\operatorname{tm}(\mathrm{n}=1 ; 1.1 \%)$ \\
\hline
\end{tabular}


However, all infants under 1 year of age, except one with serous cystadenoma had nonneoplastic pathologies, namely cystic degeneration due to intrauterine torsion, follicle cyst or simple cyst. The difference in terms of mean greatest diameter is statistically significant ( $p=0.001)$. ROC curve analysis demonstrates that size of the lesion is fairly distinctive for pathology result prediction. Defining the cut-off point as $9 \mathrm{~cm}$ has a sensitivity of $59.26 \%$ (95\% CI: 38.8-77.6) and specificity of $81.36 \%$ (95\% CI: 69.1-90.3, p=0.001). Cystic or solid nature of the lesion has a statistically significant relation with the pathologic group $(p<0.001)$. Only 2 patients with benign neoplastic cases had ovarian torsion: one with dermoid cyst and the other with serous papillary cystadenoma. Presence of torsion is higher in non-neoplastic group $(\mathrm{p}<0.001)$.

\section{c) Ovarian preservation rates with respect to} age, size, USG features and adnexal torsion (Table 3)

Differences between groups with respect to age and presence of ovarian torsion are not statistically significant $(\mathrm{p}=0.107$ and $\mathrm{p}=0.258$, respectively). When mean diameters of masses compared, the difference between groups is statistically significant $(\mathrm{p}<0.001)$. We also found that type of surgical approach is related to nature of mass $(\mathrm{p}<0.001)$.

Pathology revealed necrosis in 6 of 16 torsed oopherectomy materials in the oopherectomy group. Our institutional policy evolved in favor of ovarian-preservation in torsion cases in recent years.

\section{DISCUSSION}

Ovarian cysts are common among all pediatric age groups due to different hormonal insults. Maternal and placental hormones in infancy, release of gonadotropins by developing pituitary in prepubertal period and dysfunctional ovulation in adolescence are the causative mechanisms for ovarian cysts. ${ }^{1}$ The challenge in managing girls with ovarian cyst lies in detecting a small percentage of neoplastic lesions among all these almost physiologic ones. ${ }^{2}$ Although clinical presentation, laboratory and radiologic findings

TABLE 2. Clinical characteristics according to the patholog

\begin{tabular}{|c|c|c|c|}
\hline & $\begin{array}{c}\text { Non-neoplastic } \\
\text { Na: } 59\end{array}$ & $\begin{array}{l}\text { Neoplastic } \\
\quad \mathrm{N}: 27\end{array}$ & $p$ value \\
\hline Mean age (years) & $9.4 \pm 6.2$ & $10.4 \pm 3.8$ & 0.470 \\
\hline Mean greatest diameter $(\mathrm{cm})$ & $6.8 \pm 2.8$ & $11.2 \pm 5.9$ & 0.001 \\
\hline $\begin{array}{l}\text { Nature } \\
\text { Cystic } \\
\text { Solid } \\
\text { Cystic-solid }\end{array}$ & $\begin{array}{c}51(89.5 \%) \\
1(20 \%) \\
7(29.2 \%)\end{array}$ & $\begin{array}{c}6(10.5 \%) \\
4(80 \%) \\
17(70.8 \%)\end{array}$ & $<0.001$ \\
\hline $\begin{array}{l}\text { Ovarian torsion } \\
\text { Present } \\
\text { Absent }\end{array}$ & $\begin{array}{l}28(93.3 \%) \\
31(55.4 \%)\end{array}$ & $\begin{array}{c}2(6.7 \%) \\
25(44.6 \%)\end{array}$ & $<0.001$ \\
\hline
\end{tabular}

TABLE 3. Clinical characteristics according to the surgical approach

\begin{tabular}{|c|c|c|c|}
\hline & $\begin{array}{c}\text { Oopherectomy } \\
\mathrm{N}: 38\end{array}$ & $\begin{array}{l}\text { Ovary-sparing } \\
\text { Surgery } \\
\text { N: } 48\end{array}$ & $p$ value \\
\hline Mean age (years) & $8.6 \pm 6.04$ & $10.6 \pm 5.1$ & 0.107 \\
\hline Mean greatest diameter $(\mathrm{cm})$ & $10.2 \pm 5.3$ & $6.8 \pm 3.1$ & $<0.001$ \\
\hline $\begin{array}{l}\text { Nature } \\
\text { Cystic } \\
\text { Solid } \\
\text { Cystic-solid }\end{array}$ & $\begin{array}{c}16(28.1 \%) \\
4(80 \%) \\
18(75 \%)\end{array}$ & $\begin{array}{l}41(71.9 \%) \\
1(20 \%) \\
6(25 \%)\end{array}$ & $<0.001$ \\
\hline $\begin{array}{l}\text { Ovarian torsion } \\
\text { Present } \\
\text { Absent }\end{array}$ & $\begin{array}{l}16(53.3 \%) \\
22(39.3 \%)\end{array}$ & $\begin{array}{l}14(46.7 \%) \\
34(60.7 \%)\end{array}$ & 0.258 \\
\hline
\end{tabular}


help to differentiate, we cannot conclude the exact diagnosis before the pathology result. Decision between conservative or surgical treatment, open or minimally invasive approach and ovarianpreservation or oopherectomy are questions awaiting clinicans dealing with these conditions. More frequent occurrence of benign ovarian masses compared to malignancies in pediatric age group is the main advantage and enables a more conservative and ovarian-preservative approach.

In our study the mean age of our patients was 9.7 years in a range of $0-16$ years. This was less than the other large cohort studies. ${ }^{3-5}$ This may be attributed to a large proportion of infants under 1 year of age in our study. Widespread use of USG and prenatal surveillance enable early diagnosis at an asymptomatic age. Also, this study was conducted only by pediatric surgeons dealing with younger patients.

Tumor markers can be elevated in malignant ovarian germ cell and epithelial tumors and they can be used as a parameter in follow-up of these patients. ${ }^{6}$ There were 4 out of 25 patients with elevated tumor markers. Statistical conclusion cannnot be drawn from this data due to the small number however previous studies showed that markers are elevated in a minor group even in malignant pathologies. However, it is still recommended to test for these markers since if positive, they can be used in the postoperative follow-up. ${ }^{7}$ Elevated hormone levels and precocious puberty can be seen in sex-cord stromal tumors and autonomously functional ovarian cysts. ${ }^{1,8}$ Six patients in our study were diagnosed during the work-up of early pubertal development. All of them had benign pathologies. We may conclude that in the case of clinical symptoms of hormonally-active tumors, ovarian-preservative surgery may be justified. Magnetic resonance imaging(MRI) is excellent in ovarian pathologies regarding size, the origin of mass, presence of solid and cystic components and relation with neighbouring organs. ${ }^{9}$ However, there are major disadvantages like need for sedation in pediatric age group, high cost and not readily available in emergency cases in our hospital. We utilize USG as the first line imaging modality and cross-sectional imaging studies were added in selected cases.

Ovarian cysts caused by dysfunctional ovulation or hormonal irregulations are the usual case that we encounter during evaluation of girls in pediatric/adolescent age group but possibility of malignancy cannot be ignored. Papic et al., reviewed 150 patients in pediatric age group with an ovarian mass and evaluated predictors of malignancy. They found out that, an ovarian mass $<10 \mathrm{~cm}$, with no solid component and no positive marker has a $0.25 \%$ probability of malignancy. ${ }^{5}$ Relation of age, size, USG features and presence of adnexal torsion with respect to pathology was investigated in our study. The mean age of patients in neoplastic and nonneoplastic lesions groups and resultants were similar with no statistical difference in our study. We expected to find greater mean age in neoplastic lesions from others since most of the patients were older than 6 years old and peri-

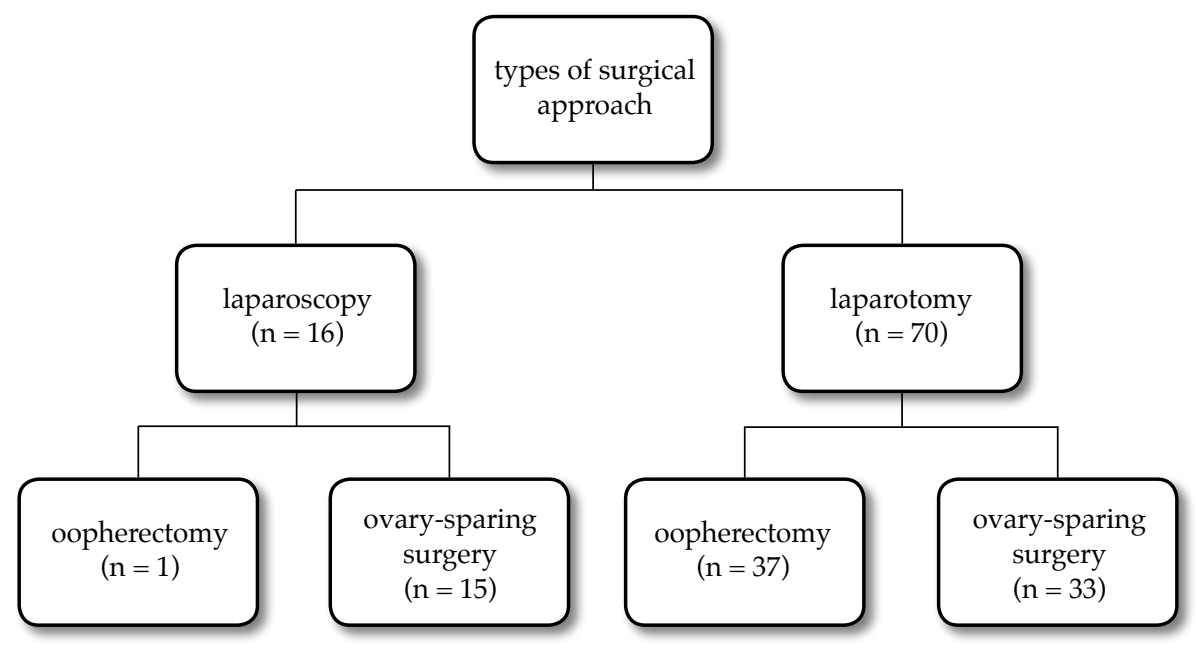


menarchal. But non-neoplastic group had a bimodel age distribution which includes infants and adolescents with respect to increased hormonal activity. For this reason, mathematical mean of this patient group equals to 9.5 years. Mean diameter of lesions was significantly greater in neoplastic group. We defined a meaningful cut-off point as $>9 \mathrm{~cm}$ for neoplastic pathologies. Cystic lesions were more frequently non-neoplastic and masses with solid components were more likely to be neoplastic. Patients with torsion had non-neoplastic pathologies in nearly most of the cases, and none with malignant diagnosis. In the light of these findings, we may infer that a purely cystic mass with a diameter $<9 \mathrm{~cm}$ with torsion is more likely to be a non-neoplastic one. A retrospective study including 502 patients support our findings and state that presence of solid component, diameter $>9 \mathrm{~cm}$ and absence of torsion are associated with an increased risk of malignancy. ${ }^{7}$ Our results were in concordance with this findings however benign and malignant cases were together grouped as neoplastic and compared with non-neoplastic pathologies in our study.

Ovarian torsion has a bimodel age distribution; one in infantile and the other in the perimenarchal period. Hormonal stimulation is blamed in etiologic theories and childhood ovarian torsion is exceedingly rare in malignant cases. However, benign tumors like mature teratoma or dermoid cyst may be seen with this phenomenon. In our series, ovarian torsion was associated with nonneoplastic lesions in $93.3 \%$ of cases. Torsion was more common among masses $<6 \mathrm{~cm}$ and of cystic nature. These findings may be explained by shallow pelvis of children which does not enable large masses to mobilize in and greater gravitational force of solid component seen in neoplastic cases. Adhesions in malignant cases may also contribute to immobility of adnexa. ${ }^{10,11}$ Current literature neither support, nor discourage routine practice of oophoropexy in pediatric age group. There is not enough evidence of oophoropexy benefit and we have considerations regarding the adenaxal organs of children and adolescent in rapidly growing phase of life may be negatively influenced by pexy sutures. ${ }^{12}$ For these reasons, we justify oophoropexy only in patients with recurrent ovarian torsion and in case of single ovary.

Laparoscopy is a safe and effective way of approaching to ovarian masses. Besides wellknown benefits of laparoscopy like small incision, early recovery and minimal risk of adhesion, it enables excellent visualisation and manipulation in small pelvis of children, ovarian-sparing surgery and minimal blood loss. ${ }^{1}$ In a study documenting 158 patients who underwent laparoscopic surgery for a gynecologic disorder, convertion to laparotomy was necessary in only 2 cases due to adhesions and giant mass which did not allow insertion of trocars. Various procedures like cystectomy, cyst drainage, detorsion, oophoropexy, oopherectomy were performed with a few and minor complications. ${ }^{13}$ We performed 16 laparoscopic surgeries since 2012 and almost all was ovarian-sparing surgeries. The reason of high percentage of laparatomy lies in the smaller amount of time since the introduction of laparoscopy to our clinic in the overall time period. This study covers the patients managed during 15 years and laparoscopy could only be used in the last 3 years of this time frame. Laparoscopy and ovarian-sparing surgery should be utilized as much as possible in pelvic pathologies since there is high risk of recurrence in even benign lesions and pelvic adhesions may cause unfavorable effects on future fertility. ${ }^{1,4,6}$ Taskinen et al., described a series of patients with mature teratoma and $23 \%$ had metachronous contralateral ovarian tumors. They performed oopherectomy in the first tumors in all patients and ovary-sparing surgery in recurrent cases. ${ }^{14}$ Presence of ovarian tissue in cyst walls of patients whom were operated by stripping technique were examined and no ovarian tissue was observed in $86.7 \%$ of cases and there were only $1 \mathrm{~mm}$ thickness ovarian tissue in 4 patients diagnosed with endometriosis. ${ }^{15}$ All these experiences and facts lead us to a more conservative, minimally invasive and ovarian preservative approach.

Ovarian cyst aspiration is one of the management options in ovarian cysts detected in prenatal or infancy period. The logic of this technique lies in the fact that almost all of the cases in this age group are of benign nature and have a tendency to resolve spontaneously. However, there is a risk of torsion and loss of ovarian tissue during the waiting period. ${ }^{16}$ Simple aspiration of cyst can be performed either by USG guidance or by assistance of laparoscopy. However, one must be sure about the origin of cyst since cystic lesions of intestinal, renal or retroperitoneal structures can be confused with ovarian cyst. There is also the risk of recurrence in simple aspiration technique. ${ }^{17}$ We prefer laparoscopy assisted cystectomy and ovarian 
preservation rather than aspiration because of aforementioned risks. Our approach for simple prenatal ovarian cyst is expectant therapy as. But in cases of huge diameters, presence of solid component, emergence of symptoms like intestinal obstruction and suspicion of cyst origin other than ovaries lead us to surgical options. A recent systematic review studying prognosis of fetal ovarian cyst found that complex and large cysts mostly represents intrauterine ovarian torsion and surgical treatment is favored earlier in follow-up of these patients. ${ }^{18}$ All patients in our prenatal group had cystic masses $>5 \mathrm{~cm}$ and 5 of them had intrauterine torsion and ovarian loss.

\section{CONCLUSIONS}

In this retrospective study, we demonstrate that size and nature of ovarian masses and presence of torsion may help in predicting the type of pathology in the preoperative period. The finding of a purely cystic mass $<9 \mathrm{~cm}$, with torsion and negative markers, orients towards a non-neoplastic mass, while the presence of solid component and absence of torsion are associated with an increased risk of malignancy. Ovarian preservation surgery should be favored as much as possible, considering future fertility and the lower incidence of malignancy in this age group. This information may add in directing surgical decision towards oopherectomy or ovariansparing surgery. Further prospective studies are needed to clearly establish an evidence-based algorithm in management of ovarian masses.

\section{REFERENCES}

1. Brandt ML, Helmrath MA. Ovarian cysts in infants and children. Semin Pediatr Surg. 2005; 14(2):78-85.

2. Brookfield KF, Cheung MC, Koniaris LG, Sola JE, Fischer AC. A population-based analysis of 1037 malignant ovarian tumors in the pediatric population. J Surg Res. 2009; 156(1):45-9.

3. Zhang M, Jiang W, Li G, Xu C. Ovarian masses in children and adolescents -an analysis of 521 clinical cases. J Pediatr
Adolesc Gynecol. 2014; 27(3):e73-7.

4. Kanizsai B, Örley J, Szigetvari I, Doszpod J. Ovarian cysts in children and adolescents: their occurrence, behavior and management. J Pediatr Adolesc Gynecol. 1998; 11(2):85-8.

5. PapicJC, Finnell SM, Slaven JE, Billmire DF, et al. Predictors of ovarian malignancy in children: overcoming clinical barriers of ovarian preservation. J Pediatr Surg. 2014; 49(1):144-8.

6. Amies Oelschlager AM, Gow KW, Morse CB, Lara-Torre E. Management of large ovarian neoplasms in pediatric and adolescent females. J Pediatr Adolesc Gynecol. 2016; 29(2):88-94.

7. Madenci AL, Levine BS, Laufer MR, Boyd TK, et al. Preoperative risk stratification of children with ovarian tumors. J Pediatr Surg. 2016; 51(9):1507-12.

8. Papanikolaou A, Michala L. Autonomous ovarian cysts in prepubertal girls. How aggressive should we be? A review of the literature. J Pediatr Adolesc Gynecol. 2015; 28(5):292-6.

9. Marro A, Allen LM, Kives SL, Moineddin R, Chavhan GB. Simulated impact of pelvic MRI in treatment planning for pediatric adnexal masses. Pediatr Radiol. 2016; 46(9):1249-57.

10. Cass DL. Ovarian torsion. Semin Pediatr Surg. 2005; 14(2):86-92.

11. Ngo AV, Otjen JP, Parisi MT, Ferguson MR, et al. Pediatric ovarian torsion: a pictorial review. Pediatr Radiol. 2015; 45(12):1845-55.

12. Dasgupta R, Renaud E, Goldin AB, Baird R, et al. Ovarian torsion in pediatric and adolescent patients: A systematic review. J Pediatr Surg. 2018; 53(7):1387-91.

13. Rieger MM, Santos XM, Sangi-Haghpeykar H, Bercaw JL, Dietrich JE. Laparoscopic outcomes for pelvic pathology in children and adolescents among patients presenting to the pediatric and adolescent gynecology service. J Pediatr Adolesc Gynecol. 2015; 28(3):157-62.

14. Taskinen S, Urtane A, Fagerholm R, Lohi J, Taskinen M. Metachronous benign ovarian tumors are not uncommon in children. J Pediatr Surg. 2014; 49(4):543-5.

15. Palmara V, SturleseE, Romeo C, Arena F, et al. Morphologic study of the residual ovarian tissue removed by laparoscopy or laparotomy in adolescents with benign ovarian cysts. J Pediatr Surg. 2012; 47(3):577-80.

16. Cho MJ, Kim DY, Kim SC. Ovarian Cyst Aspiration in the Neonate: Minimally Invasive Surgery. J Pediatr Adolesc Gynecol. 2015; 28(5):348-53.

17. Rialon KL, Akinkuotu A, Fahy AS, Shelmerdine S, et al. Management of ovarian lesions diagnosed during infancy. J Pediatr Surg. 2019. [Epub ahead of print].

18. Bascietto F, Liberati M, Marrone L, Khalil A, et al. Outcome of fetal ovarian cysts diagnosed on prenatal ultrasound examination: systematic review and meta-analysis. Ultrasound Obstet Gynecol. 2017; 50(1):20-31. 